RUNNING HEAD: HBT \& COVID-19 VACCINE

Integrating health behavior theories to predict COVID-19 vaccines uptake intent among the American public

Haoran $\mathrm{Chu}^{1}$, and Sixiao Liu ${ }^{2}$

${ }^{1}$ Department of Public Relations, College of Media and Communication, Texas Tech University.

Address: $300315^{\text {th }}$ Street, Lubbock TX 79409, USA.

Email: haoran.chu@,ttu.edu

${ }^{2}$ Department of Communication, University at Buffalo.

Address: 426 Baldy Hall, University at Buffalo North Campus, Buffalo NY 14260, USA.

Email: sixiaoli@buffalo.edu 


\title{
Integrating health behavior theories to predict COVID-19 vaccines uptake intent among the American public
}

\begin{abstract}
Wide uptake of coronavirus disease 2019 (COVID-19) vaccines is key to containing the ongoing pandemic. Integrating constructs from three prominent health behavior theories (HBT) including the extended parallel process model (EPPM), the health belief model (HBM) and the theory of planned behavior (TPB), the current study seeks to identify sociopsychological factors that influence American public's COVID-19 vaccines uptake intent. An online survey was delivered to a national sample $(N=934)$ with demographic composition similar to the U.S. population. This study assessed the influences of risk perception and fear associated with COVID-19, beliefs about and attitudes toward COVID-19 vaccines, self-efficacy, social and psychological contexts, and demographic characteristics on people's intention to get COVID-19 vaccines. Most respondents intended to get a COVID-19 vaccine once it is available. However, participants tended to underestimate their risks of contracting COVID-19, which may prevent them from getting vaccinated. Exposure to COVID-19 infections and deaths led to higher uptake intent via the mediation of fear. Concerns about the safety of COVID-19 vaccines negatively influenced vaccination intention, while perceived community benefits of the vaccines were positively associated with vaccination intention. Positive attitudes toward vaccines in general and recent vaccine history were also positively linked to COVID-19 vaccines uptake intent. This study attests the effectiveness of HBT constructs in predicting COVID-19 vaccines uptake intent. The results point to the importance of fostering confidence in vaccine safety in interventions seeking to promote COVID-19 vaccines uptake.
\end{abstract}

Keywords: COVID-19; COVID-19 vaccines; HBM; TPB; EPPM 


\section{Background}

The coronavirus disease-2019 (COVID-19) pandemic has caused catastrophic damages to societies across the world (World Health Organization [WHO], 2020). As of December 2020, more than 68 million cases of COVID-19 infections and more than 1.5 million deaths caused by the disease have been reported worldwide (WHO, 2020). However, as effective treatment of the disease is yet to be available, public health authorities are relying on preventive measures to curb the spread of COVID-19 (Bloom et al., 2020; Schoch-Spana et al., 2020).

One of the most effective preventive measures to contain the spread of infectious diseases is vaccine (Centers for Disease Control and Prevention [CDC], 2011). Effective vaccination not only foster individual immunity against vaccine preventable diseases (VPD), but also protect those unable to get vaccines through "herd immunity" (Betsch et al., 2017). However, despite its effectiveness in preventing infectious diseases, vaccine hesitancy, which describes attitudes and behaviors surrounding vaccine delay and refusal, is on the rise in many societies, leading to the recent re-emergence of VPDs such as measles (Dubé et al., 2013; Quinn et al., 2019). Though governments and public health institutions worldwide have been anxiously expecting vaccines to control the ongoing pandemic, a recent poll shows that almost half of American adults are reluctant to get COVID-19 vaccines (Tyson et al., 2020). To develop effective interventions that promote wide acceptance of COVID-19 vaccines once they are available, it is critical to identify factors that influence people's COVID-19 vaccines uptake intent.

\section{Health Behavior Theories}

Decades of research in health psychology have generated multiple health behavior theories (HBT) that identify sociopsychological factors influencing individual's health decision- 
making (Noar \& Zimmerman, 2005). Studies on vaccines uptake behavior and behavioral intention ranging from childhood vaccines (Chen et al., 2011) to HPV vaccines (Gerend \& Shepherd, 2012) also benefited tremendously from HBTs. Though HBTs often include different constructs, most of them focus on several similar sets of factors that predict the initiation and maintenance of health behaviors (Noar \& Zimmerman, 2005; Weinstein, 1993).

Correspondingly, researchers have long rallied for the comparative examination, refinement, and integrations of different HBTs (Gerend \& Shepherd, 2012; Noar \& Zimmerman, 2005;

Weinstein, 1993). In the meantime, the novel challenges brought about by the COVID-19 pandemic also necessitate a comprehensive investigation of sociopsychological factors that predict people's intention to get the COVID-19 vaccines once they are widely available.

To address these two goals, that are to comparatively analyze the efficacy of different HBTs and to identify sociopsychological factors that determine COVID-19 vaccines uptake intent, this study examines five areas of constructs that are commonly utilized in HBTs and seeks to examine their influences on COVID-19 vaccines uptake intent. Based on three prominent HBTs including the health belief model (HBM) (Becker, 1974), the theory of planned behavior (TPB) (Azjen, 1980), and extended parallel process model (EPPM) (Witte, 1994), we focus on factors including risk perception and fear associated with COVID-19, attitudes and beliefs related to COVID-19 vaccines, self-efficacy, social and psychological contexts, and demographics. Notably, as effective and clinically tested COVID-19 vaccines are yet to be widely available in the United States when this study was conducted, we focus on the intention to get COVID-19 vaccines due to its strong association with the actual uptake behavior (Azjen, 1980; Gerend \& Shepherd, 2012).

\subsection{COVID-19 risk perception and fear}


Vaccination, like many health behaviors, is adopted to prevent negative health consequences such as contracting and suffering from VPDs (Brien et al., 2012). Preventive behavior is necessary when not performing it would lead to adverse consequences. It is thus reasonable to believe that the extent to which people believe such threat is severe and likely to affect them should also predict their intention to adopt the preventive behavior (Weinstein, 1993). HBM and EPPM measures people's perception of health risks with similar constructs, including perceived severity of the negative health consequences and perceived susceptibility to adverse health impacts (Noar \& Zimmerman, 2005). Based on the expectancy-value framework, some research models risk perception as the product term of perceived severity and perceived susceptibility (Rimal \& Real, 2003). However, HBM and EPPM research often analyzes perceived severity of and susceptibility to VPDs as separate predictors without modeling their interaction effect (Gerend \& Shepherd, 2012; Janz \& Becker, 1984; Witte, 1994).

In addition to risk perception, HBTs also suggest that affective response to health risks motivate actions to overcome their adverse effects (Abril et al., 2017; Witte, 1994). Fear as a negative emotion was particularly relevant to people's adoption of health behaviors (Liu et al., 2019). It is worth noting that though the lack of fear often make health behavior less desirable, too much fear could also prevent action as people may just disregard the threats to avoid feeling overwhelmed (Witte, 1994). Therefore, a moderate level of fear often motivates preventive actions such as getting vaccinated or screened for diseases (Maloney, Lapinski, \& Witte, 2011). In the context of COVID-19 vaccines uptake, the large number of infections and casualties may have led to heightened risk perception and strong fear (Dryhurst et al., 2020). However, the prolonged pandemic along with the comparatively lower mortality rates among certain populations (e.g., younger populations) may also lead to fatigue of practicing preventive 
measures such as social distancing. The overoptimism of their invulnerability to the disease may also lower their risk perception and fear. For instance, despite repeated warnings issued by public health authorities, millions of Americans travelled during the holiday season (Groves, 2020). It is possible that for many individuals, COVID-19 may not be a very intimidating threat as experts suggest. Therefore, it is reasonable to expect that some may also hesitate to get the vaccines due to lower risk perception and fear. To investigate such possibility, we hypothesize that:

H1. Risk perception (i.e., perceived severity of and susceptibility to COVID-19) and fear will be positively associated with COVID-19 vaccines uptake intent.

\subsection{COVID-19 vaccines attitudes and beliefs}

In addition to risk perception and fear, HBTs argue that attitudes toward and beliefs about the health behaviors also shape people's intention to adopt them (Gerend \& Shepherd, 2012; Noar \& Zimmerman, 2005). Such attitudes and beliefs are operationalized differently across models, such as perceived benefits and barriers in HBM, response efficacy in EPPM, and positive or negative behavioral beliefs in TPB (Noar \& Zimmerman, 2005). However, they can also be categorized into two subgroups, including perceived benefits and barriers or costs of health behaviors, respectively capturing the positive and negative outlooks associated with the adoption of health behaviors. Notably, different from severity and susceptibility perception, which correspond to the value and expectancy components of disease threat evaluation, perceptions of a health behavior (e.g., perceived benefit, response efficacy) are conceptualized as beliefs in the health behavior's ability to reduce disease threat in both severity and susceptibility (Janz \& Becker, 1984; Witte, 1994). For instance, a vaccine may be perceived as beneficial and efficacious because it reduces individual susceptibility to its related VPD, while a painkiller may be perceived as beneficial because it relieved the severity of physical pains. In vaccine research, 
perceived benefits of vaccines (e.g., reduced disease threat) were found to be strong predictors of vaccination intention, while perceived cost or barriers (e.g., monetary cost, effort exertion) of getting vaccinated were negatively associated with uptake intent (e.g., influenza vaccine (Shahrabani \& Benzion, 2012), HPV vaccines (Gerend et al., 2013), Zika Vaccine (Guidry et al., 2019), Childhood vaccine (Smith et al., 2011).

In the context of COVID-19 vaccines, we focus on five interrelated attitudes and beliefs associated with the vaccines and their impacts on vaccines uptake intent, including perceived benefits of getting COVID-19 vaccines for self and communities, perceived barriers of getting COVID-19 vaccines (i.e., safety and cost concerns), and attitudes towards to getting COVID-19 vaccines. We ask if these factors would be differently linked to COVID-19 vaccines uptake intent (RQ1a). Notably, perceived benefits and barriers are conceptually similar to positive and negative behavioral beliefs specified in TPB (Weinstein, 1993). The theory suggests that such beliefs shape people's attitudes toward the behavior, which subsequently influence behavioral intent (Noar \& Zimmerman, 2005). We thus examine if attitudes toward COVID-19 vaccines mediates perceived benefits and barriers are associated with COVID-19 vaccines uptake intent (RQ1b).

\subsection{Self-efficacy}

Knowing that health behaviors such as getting vaccinated help prevent negative consequences may not be enough to motivate behavioral adoption (Noar \& Zimmerman, 2005; Weinstein, 1993). HBTs argue that feeling capable of executing such behaviors are also key to behavioral intent and actual behaviors (Maloney et al., 2011; Petrovic et al., 2011). Such perceived capability is conceptualized as perceived behavioral control in TPB and self-efficacy in EPPM (Noar \& Zimmerman, 2005). Observably, recent TPB studies often operationalize 
perceived behavioral control as self-efficacy due to their similarities (Ajzen, 2002; Noar \& Zimmerman, 2005). Research shows that self-efficacy is positively related to vaccines uptake (e.g. influenza vaccines (Gargano et al., 2011), HPV vaccines (Brewer \& Fazekas, 2007)). Therefore, we hypothesize that self-efficacy is positively associated with people's intention to get COVID-19 vaccines (H2).

\subsection{Social and psychological context}

We extract three sets of contextual factors from HBTs and vaccine research and analyze their relations with COVID-19 vaccines uptake intent, including subjective norms (TPB; Catalano et al., 2017), cues to action (HBM; Gerend \& Shepherd, 2012), and baseline vaccine hesitancy (Dubé et al., 2013). Subjective norms include injunctive norms, which are the approvals of health behaviors by people important to the health decision-maker (i.e. people important to me think that I should get COVID-19 vaccines), and descriptive norms, which are perception of important others' behaviors (i.e. people important to me will get COVID-19 vaccines) (Fishbein \& Ajzen, 2011). TPB research shows that both descriptive and injunctive norms are positively related to vaccines uptake (Visser et al., 2018; Xiao \& Borah, 2020).

Cues to action is an integral construct of HBM which specifies motives and hints of actions embedded in one's surrounding environment such as doctor's recommendations (Gerend \& Shepherd, 2012). Additionally, research also shows that disease exposure or knowledge of VPDs are positive predictors of vaccines uptake (Brewer \& Fazekas, 2007). Specifically, witnessing or knowing someone contracting a VPD often signals the vulnerability of oneself which may thus necessitate need to get vaccinated (Qian \& Li, 2020). Having more knowledge about diseases and vaccines also help people understand the importance of vaccination and increase their uptake intent (MacDonald, 2015). In the COVID-19 context, it is thus possible that 
exposure to COVID-19 cases such having family or friends tested positive for COVID-19 may function as a behavioral cue (Dubé et al., 2013). Further, serving as an essential worker during the COVID-19 lockdowns may also be a cue to action. On the one hand, essential workers may be more susceptible to the disease due to the risk of exposure, while on the other, public health officials have called for earlier vaccination for this group of individuals (Bloom et al., 2020; Schoch-Spana et al., 2020).

The last set of contextual factors include baseline vaccine hesitancy, which describes the attitudes and behaviors surrounding people's decision to delay and refuse vaccination (Dubé et al., 2013). Vaccine hesitancy is often operationalized as attitudes toward vaccines or past vaccination behavior (Dubé et al., 2013; Quinn et al., 2019). Research shows that positive attitudes toward vaccines in general are positively associated with people's intent to get specific vaccines (Dubé et al., 2013; MacDonald, 2015), and individuals who have received vaccines recently are more likely to get other vaccines (Marlow et al., 2007).

In addition to their direct effects, risk perception, fear, self-efficacy, and beliefs about COVID-19 vaccines may also mediate contextual factors' influence on vaccines uptake intent. First, social cognitive theory suggests that people learn not only by directly interacting with the environment, but also by vicariously observing other's actions (Bandura \& Walters, 1977). Therefore, socially important others' beliefs and behaviors related to vaccines may also influence people's evaluation of the vaccine and their confidence in getting vaccinated (Gerend \& Shepherd, 2012). Supporting such argument, Stout et al. (2020) found that self-efficacy mediated social norm's influence on people's intention to get HPV vaccines. In the context of COVID-19 vaccines, it is likely that subjective norms may also influence self-efficacy, which may further mediate its influence on uptake intent. Second, as COVID-19 vaccines are yet to be available, 
people's perception of the vaccines may be influenced by their existing attitudes towards other vaccines (Dubé et al., 2013). For instance, Quinn et al. (2019) found that attitudes toward vaccine in general and vaccination history are all positively associated with people's attitudes toward a new flu vaccine. It is thus reasonable to argue that attitudes toward COVID-19 vaccines may also mediate baseline vaccine-hesitancy's influences on vaccines uptake intent. Third, exposure to COVID-19 cases and serving as essential workers may also lead to higher chance of contracting the disease and elevated perception of its danger. Such contextual cues may thus influence vaccines uptake intent because of the increased severity and susceptibility perception. Lastly, recent vaccination history may also boost self-efficacy which would lead to increased uptake intent, as successful past experiences of adopting health behaviors are often positively related to self-efficacy (Bandura \& Walters, 1977).

RQ2. Are contextual factors including subjective norms, cues to actions, and vaccine hesitancy directly and indirectly associated with people's COVID-19 vaccines uptake intent?

\subsection{Demographic factors}

Lastly, demographic factors are also associated with vaccines uptake intent. Due to historical and cultural reasons, minorities in the United States are often reluctant to receive novel medical treatment (Cates et al., 2009; Quinn et al., 2017). Gender difference in vaccination behavior was also observed in some studies, but the patterns of such difference vary across contexts (Nan, 2012). Older adults and individuals with higher education and income often show higher vaccination intention (Chapman \& Coups, 1999; Kumar et al., 2012; Larson et al., 2014). We therefore ask our last research question:

RQ3: How are demographic characteristics associated with COVID-19 vaccines uptake intent? 


\subsection{Theoretical framework}

Figure 1 summarizes the theoretical framework presented above. As illustrated earlier, we followed conventional operationalization of EPPM (Witte, 1994), HBM (Janz \& Becker, 1984), and TPB (Gerend \& Shepherd, 2012) constructs by modeling the effects of these factors additively. However, it is notable that some research operationalizes risk perception as the product term of severity and susceptibility perception. We tested such possibility and report the result of the multiplicative model in the Appendix (Supplementary Table 1). Notably, the interaction term did not correlate significantly with vaccine uptake intent and the increase in the model-explained percentage of variance in uptake intent is $0.7 \%$.

\section{Method}

Upon approval from the Institutional Review Board at the lead author's institution, a survey hosted on Qualtrics.com was distributed to a group of research participants recruited on Prolific.co, a crowdsourcing platform for researchers and participants (Palan \& Schitter, 2018). After providing informed consent to participate in the study, respondents answered the survey questions. The median completion time of the survey questionnaire was approximately 12 minutes.

\subsection{Data Collection}

Data collection was completed during early September 2020. More than half year into the pandemic, several vaccines were under second and third-stage trials during the data collection period in the United States and United Kingdom, and countries around the world have ramped up their COVID-19 testing (Llyod, 2020). The increase in new cases and mortality associated with 
COVID-19 were also less rapid during the summer of 2020 as compared to the earlier months (WHO, 2020).

A sample of participants with demographics similar to the United States population was recruited on Prolific.co. The platform uses algorithms to recruit participants based on their age, gender, and race to generate a nationally representative sample. 1,043 Individuals on the platform opened the survey, with 1,027 provided informed consent to participate in the survey. Among them, 1001 participants completed the survey questionnaire and was rewarded 2.00 U.S. dollars for their time. 54 Participants (5.4\%) failed one or two attention check questions embedded in the survey and their responses were removed in the subsequent analyses. Responses from 13 participants who indicated that they were tested positive for COVID-19 and/or COVID19 antibody were also dropped, resulting in a final sample of 934 participants. The sample size was determined by conventional heuristic which suggests a case-indicator ratio of 5:1 (5 participants per each indicator; Bentler \& Chou, 1987) and accounted for attrition. Power analysis indicates that a sample of at least 704 cases is sufficient in detecting relatively small effect size (Cohen's $d=0.2, \alpha=0.05$, Power $=0.95)$ in the structural model specified in Figure 1 (Westland, 2010). This study was not pre-registered, and there was no missing observation in the final dataset. No imputation is thus utilized (Sidi \& Harel, 2018).

\subsection{Instruments}

HBT and vaccine research constructs including perceived severity of and susceptibility to COVID-19, fear of COVID-19 (Brewer er al., 2007; Liu et al., 2019), perceived individual benefits of COVID-19 vaccines (Brabin et al., 2006; Gerend \& Shepherd, 2012), perceived community benefits of COVID-19 vaccines, perceived barriers to getting COVID-19 vaccines including safety concerns and cost concerns (Gerend et al., 2013), subjective norms, baseline 
vaccine hesitancy including attitudes toward vaccines in general and recent vaccination history, cues to actions (i.e. exposure to COVID-19 cases and serving as essential workers during the COVID-19 lockdowns), and demographics were measured in the survey. Measurement items, reliability indices, means, and standard deviations of the measurement instruments are presented in Table 1. Scales were adopted from existing research if available.

\subsection{Analysis}

Q-Q plot was generated in R to assess multivariate normality of the measurement items (Korkmaz et al., 2014). Upon inspection, the data failed to achieve multivariate normality. To account for non-normality, all measurement and path models were analyzed with maximum likelihood estimation with robust standard errors (Muthén \& Muthén, 2018). Indirect effect models were estimated with maximum likelihood estimation with bootstrapped sub-samples $(N=$ 5,000) (Muthén \& Muthén, 2018).

Following Kline's recommendations (Kline, 2015), we first inspected the measurement model of the latent factors. The model has achieved satisfactory fit based on conventional criteria $\left(\right.$ Hu \& Bentler, 1999; Kline, 2015) $\left(\right.$ Adjusted $\chi^{2}(d f=636)=1207.693, \mathrm{CFI}=0.981, \mathrm{TLI}=$ $0.977, \mathrm{RMSEA}=0.031,95 \%$ confidence interval of RMSEA $=[0.028,0.034], \mathrm{SRMR}=0.039)$.

A path model based on the theoretical framework illustrated in Figure 1 was further estimated. Latent HBT constructs without directional effects specified between each other were allowed to covary to account for common method and conceptual covariances. The path model also fits the data well (Adjusted $\chi^{2}(d f=1127)=2314.812, \mathrm{CFI}=0.965, \mathrm{TLI}=0.962, \mathrm{RMSEA}=$ $0.034,95 \%$ confidence interval of $\operatorname{RMSEA}=[0.032,0.036], \mathrm{SRMR}=0.054)$ based on conventional criteria (Hu \& Bentler, 1999; Kline, 2015). 


\section{Results}

Path coefficient estimates are reported in Figure 2 and Table 2-4. Indirect effects are reported in Table 5 . The path model was able to predict $82.1 \%$ of variance in participants' COVID-19 vaccines uptake intent $\left(R^{2}=.821\right)$.

\subsection{Demographics}

The average age of our participants is 46.01 years $(S D=16.17)$. The majority of participants identified as female ( $n=468,50.1 \%)$, followed by male $(n=455,48.7 \%)$ and other gender $(n=11,1.2 \%)$. Approximately three quarters of participants identified as non-Hispanic White or Caucasian $(n=698,74.7 \%)$, followed by Black or African American $(n=114,12.2 \%)$, Hispanic or Latino $(n=34,3.6 \%)$, Asian, Pacific Islander or Native American $(n=68,7.3 \%)$, and other racial groups ( $n=20,2.1 \%$ ). The median education level is 4-year college degree, and the median household income falls in the bracket between US\$ 50,000 and 74,999. Participants' geographical locations are illustrated in Figure 3. RQ3 asks about demographic factors' association with COVID-19 vaccines uptake intent. However, no significant effect of the demographic variables was identified after controlling for the HBT constructs.

\subsection{COVID-19 risk perception and fear}

In general, our participants perceived COVID-19 as a very severe health risk. However, their average rating of perceived susceptibility to COVID-19 was lower than the average rating of perceived severity on the similar 5-point scale (paired-sample $t(933)=28.70, p<.001$ ). The average rating for fear was also below the scale mid-point (Table 1). Corresponding to $\mathbf{H 1}$ which argues that perceived severity of and susceptibility to COVID-19 and fear would influence COVID-19 vaccines uptake intent, only fear was positively associated with COVID-19 vaccines 
uptake intent in the structural model. However, it is also notable that perceived susceptibility's association with vaccines uptake intent was approaching statistical significance $(B=0.114, S E=$ $0.062, p=0.064)$ and such effect was statistically significant in the multiplicative model $(B=$ 0.124, $S E=0.061, p=0.043$; Supplementary Table 1).

\subsection{COVID-19 vaccines attitudes and beliefs}

Participants on average showed positive attitudes toward COVID-19 vaccines. In general, they also perceived COVID-19 vaccines as beneficial to both themselves and their communities (i.e., average ratings above scale mid-point). In terms of possible barriers to getting vaccinated, participants rated concerns about vaccine safety (e.g. not enough research on the vaccines, side effect) as more important reasons that may prevent vaccination than cost concerns (e.g. out-ofpocket cost, insurance coverage; paired-sample $t(933)=28.36, p<.001)$. In response to RQ1a which enquires about COVID-19 vaccines-related attitudes and beliefs' direct impacts on uptake intent, we found that perceived community benefits of and attitudes toward COVID-19 vaccines were positively associated with intention to get the vaccines. However, stronger safety concerns were related to lower uptake intent. In addition to their direct effects, specific beliefs about COVID-19 vaccines' benefits and barriers also indirectly influenced uptake intent (RQ1b). Attitudes toward COVID-19 vaccines mediated perceived community benefits and safety concerns' association with uptake intent, but the former was positive while the latter was negative. Interestingly, attitudes toward COVID-19 vaccines also mediated cost concerns' positive relationship with vaccines uptake. Specifically, increased concerns of COVID-19 vaccines cost were positively correlated with attitudes toward the vaccines, which is positively associated with vaccines uptake intent.

\subsection{Self-efficacy}


Our respondents generally believed that they will be able to get COVID-19 vaccines to prevent contracting COVID-19. We also analyzed if self-efficacy is related to vaccines uptake intent (H2). Different from existing research findings, no significant relationship was identified between self-efficacy and intention to get vaccinated for COVID-19.

\subsection{Contextual cues and baseline vaccine hesitancy}

More than half of our final sample have had some experiences with COVID-19. Particularly, more than one third of participants knew someone who was tested positive for COVID-19 and more than $20 \%$ of participants reported having family or close friends tested positive for COVID-19. Further, more than one hundred respondents indicated that someone they knew died due to COVID-19, again showing the dire situation faced by the American public during the pandemic. Serving as essential workers may also serve as a cue to action. About $20 \%$ of our respondents indicated that they worked as an essential worker during the COVID-19 lockdowns. As for subjective norms, our participants in general believed that people like them or are important to them will get the COVID-19 vaccines (i.e. descriptive norms) and want them to get vaccinated for the disease (i.e. injunctive norms). In terms of baseline vaccine hesitancy operationalized as attitudes toward vaccine in general and recent vaccination experience, our participants largely thought of vaccines positively and more than half of them received some vaccines in the past 18 months before they took the survey.

RQ2 asks if these contextual cues and baseline vaccine hesitancy are directly and indirectly associated with people's COVID-19 vaccines uptake intent. We found that having positive baseline attitudes toward vaccines in general and received some vaccines recently were positive predictors of COVID-19 vaccines uptake intent. Further, believing that others will get vaccinated for COVID-19 (i.e. descriptive norms) was also positively associated with 
vaccination intentions. Though exposure to COVID-19 cases (e.g. knowing someone tested positive) was not directly linked to vaccines uptake intent, fear mediated COVID-19 exposure's positive relationship with vaccination intention. Specifically, knowing someone who has been tested positive for COVID-19 or died due to contracting the disease is associated with stronger fear, which correlates with higher vaccination intention. Additionally, perceived benefits of COVID-19 vaccines and attitude toward COVID-19 vaccines also mediated general vaccines attitude's positive association with uptake intent.

\section{Discussion}

The current study integrates factors from three prominent HBTs including EPPM, HBM, and TPB to investigate sociopsychological factors associated with American public's intentions to get COVID-19 vaccines that were under rapid development when the study was conducted. In general, our participants indicated that they are likely to get vaccinated for COVID-19 once the vaccines are available. This finding is encouraging as massive vaccination is key to controlling the spread of COVID-19 (Schoch-Spana et al., 2020). However, it is also notable that approximately $20 \%$ of participants reported some extent of unwillingness to get the vaccines (average uptake intent score below the scale mid-point). Considering that herd immunity against VPDs sometimes require an immunization rate of $70 \%$ to $90 \%$ (Bloom et al., 2020), and the immunity against COVID-19 developed by vaccination may not last as long as immunity for other diseases (Bloom et al., 2020), critical efforts are needed to design and implement interventions that could effectively promote vaccination, especially among populations reluctant to get the vaccines.

\subsection{COVID-19 risk perception and fear}


Participants in general perceived COVID-19 as a severe health risk. However, they tend not to feel very vulnerable to and afraid of the threats posed by the disease. Such findings showed that people are generally optimistic about their own risks of suffering from COVID-19, which is consistent with the optimistic bias observed in earlier research (Dryhurst et al., 2020; Schoch-Spana et al., 2020). Notably, as data for this study were collected before the second wave of rapid increases in COVID-19 cases and mortality in the United States (WHO, 2020), public perception of COVID-19 risks may have increased in the subsequent months due to the surge of COVID-19 infections and deaths. It is also worth noting that public attention has been drawn to civil unrests across the United States and the upcoming presidential election during such time, which may have influenced the perceived salience of COVID-19 risks.

Fear was positively associated with vaccines uptake intent for two possible reasons. First, fear as emotional response may arise from cognitive appraisals of COVID-19 and served a more immediate predictor of uptake intent than susceptibility perception (Slovic et al., 2004). Second, we may fail to capture any effect of severity perception due to its limited variance (i.e. ceiling effect). In summary, it is reasonable to suggest that the general public has recognized the severity of COVID-19, but the underestimation of their own risks of contracting and suffering from the disease may prevent them from getting vaccinated. Campaigns to promote COVID-19 vaccines should consider highlighting personal risks to the disease and using fear appeal messages when communicating to populations that are less susceptible to COVID-19 (e.g. younger population).

\subsection{COVID-19 vaccines attitudes and beliefs}

Results from the current study indicate that the American public held a generally positive view toward COVID-19 vaccines. They considered the vaccines as beneficial not only to themselves but also to their communities. However, the accelerated development of the vaccines, 
especially in comparison to earlier vaccines, may have led to heightened safety concerns (Schoch-Spana et al., 2020; Tyson et al., 2020). Cost concerns, on the other hand, was not a serious barrier to people's vaccination intentions. Notably, cost concerns such as out-of-pocket cost and insurance coverage of the COVID-19 vaccines may seem trivial when this study was conducted as the pandemic was still causing disastrous impacts and people were yearning for an effective vaccine. However, such triviality may at the same time reflect the conditional influence of different behavior barriers (Gerend et al., 2013). Specifically, as COVID-19 vaccines were yet to be widely available in September 2020, concrete considerations of costs may be less important to people's vaccination decision-making compared to safety concerns (Gerend et al., 2013).

More importantly, we found that perceived community benefit of COVID-19 vaccines was positively associated with uptake intent both directly and indirectly through the mediation of attitudes toward the COVID-19 vaccines. Differently, concerns about the safety of COVID-19 vaccines were negatively associated with uptake intent, directly and indirectly through the mediation of attitudes toward the COVID-19 vaccines. Therefore, public health campaigns aiming at increasing the vaccination rate should address the community benefits of COVID-19 vaccines (e.g. they protect not only yourself, but also vulnerable individuals in your neighborhood), while also maintain high level of transparency regarding the vaccines' safety and effectiveness. Notably, we also found that cost concerns are positively associated with intention to get vaccinated for COVID-19, and the relationship is mediated by attitudes toward the COVID-19 vaccines. Such pattern is consistent with Gerend et al.'s (2013) finding where barriers involving practical concerns (e.g., costs) are often positively linked to behavioral intention, as those intended to get vaccinated often think about the behavior in more concrete 
terms. Therefore, providing the vaccines at a reasonable cost or for free may help increase vaccination rates, especially among people who intend to get the vaccines.

\subsection{Self-efficacy}

We found that people in general are confident in their ability and resources to get the COVID-19 vaccines when they become available. However, self-efficacy was not significantly associated with uptake intent. We suspect that the absence of a significant relationship may be due to the fact that these vaccines are still not available. As the transtheoretical model (TTM) of behavioral change suggests, difference in self-efficacy tend to develop at later stages of people's health decision-making (Lipschitz et al., 2013). Limited variance in self-efficacy may have thus prevented us from identifying its relationship with COVID-19 vaccines uptake intent. We recommend future research to test this construct's effects on uptake intent and behavior when the vaccines become available. Notably, injunctive and descriptive norms were differently associated self-efficacy. Believing that important others will get the vaccines (i.e., descriptive norms) is positively related to self-efficacy, whereas the relationships is negative between beliefs that important others would want oneself to get vaccinated (i.e., injunctive norms) and self-efficacy. Such difference may emerge as people learn from others' behaviors (Bandura \& Walters, 1977), but too much preaching may also lead to psychological reactance.

\subsection{Contextual cues and baseline vaccine hesitancy}

Our findings confirm the widespread of COVID-19 cases and mortalities reported elsewhere (Dryhurst et al., 2020; WHO, 2020). The majority of our sample had some first-hand experience with the disease, which is related to heightened fear and increased intention to get vaccinated for COVID-19. Such finding may indicate that exposure to COVID-19 cases 
countered the negative influences of overoptimism on vaccination intention. Therefore, campaigns encouraging vaccination against COVID-19 may stress the personal relevance of COVID-19 as a means to overcome overoptimism and stipulate vaccination intention. Indeed, as more than 60 million people across the world have contracted the disease as of December 2020 (WHO, 2020), disease exposure may serve as an effective cue to action for many people. It is also encouraging to see that most of our participants held positive views toward vaccines and considered getting COVID-19 vaccines a socially sanctioned behavior. Moreover, baseline attitudes toward vaccines in general and the vaccine-friendly norms were also conducive to COVID-19 vaccines uptake intent. Therefore, promotion of COVID-19 vaccines should be considered as an integral part of the long-term efforts to reduce vaccine hesitancy (Dubé et al., 2013). It is necessary to not only foster acceptance of the COVID-19 vaccines, but also cultivate an enduring trust in vaccines and other health measures.

\subsection{Implications}

Findings from the current study point to several practical implications for health campaigns and education aiming at promoting COVID-19 vaccination. First and foremost, the prolonged struggle caused by the pandemic may have led to fatigue of practicing preventive measures and overoptimism in people's vulnerability to the disease. Therefore, it is important for public health communicators to emphasize the personal relevance of the disease and foster adequate amount of fear among the public, which are conducive to COVID-19 vaccines uptake intent. Second, campaigns need to address the safety concerns associated with COVID-19 vaccines. It is imperative to remain transparent and truthful in communications about the vaccines, as public confidence in the vaccines' safety and effectiveness are closely associated with COVID-19 vaccines uptake. Third, the vaccines' community benefits (e.g. protecting the 
vulnerable others) need to be highlighted in campaigns and education materials. This is especially true considering that some populations such as young people may think of themselves as less susceptible to the disease and less in need of a vaccine. Informing this group about the collective benefits of COVID-19 vaccines may encourage them to actually get vaccinated. Lastly, as the results indicate, COVID-19 vaccine promotion needs to be considered as an integral part of the prolonged effort to cultivate vaccine acceptance in our societies. Long-term investment in the education of vaccines and other health measures needs to be sustained to maximize the effectiveness of preventive medicines.

\subsection{Limitations}

This study also has some limitations. First, the online sample may have limited the generalizability of some of our findings. Future research should consider recruiting a more diverse sample and re-examine the relationships reported here. Second, as the COVID-19 vaccines are yet to be widely available when this study was conducted, actual vaccines uptake was not measured. Though behavioral intention and actual behaviors tend to correlate highly (Azjen, 1980), we recommend future research to test the current framework at later stage of the pandemic (i.e. when the vaccines are widely available) or with other vaccines and health behaviors.

\section{Conclusion}

Integrating findings from prominent health behavior theories, this study offers a timely overview of sociopsychological factors that are related to American public's intention to get vaccinated for COVID-19. We found that despite recognizing the severity of the disease, people felt less susceptible to and afraid of its negative consequences. Such overoptimism may also 
prevent wide acceptance of COVID-19 vaccines when they become widely available. Further, safety concerns, which may be attributed to the accelerated development and approval of vaccines, is negatively associated with vaccination intention. Campaigns promoting COVID-19 vaccines thus need to remain transparent and truthful to gain public confidence in the vaccines' safety and effectiveness. Lastly, public acceptance of COVID-19 vaccines is largely influenced by their perceptions of vaccines in general, which confirms the importance of sustained efforts in cultivating vaccine acceptance among the public. 


\section{Reference}

Abril, E.P., Szczypka, G., \& Emery, S.L. (2017). LMFAO! Humor as a response to fear: Decomposing fear control within the extended parallel process model. Journal of broadcasting \& electronic media, 61, 126-143.

Ajzen, I. (2002). Perceived behavioral control, self-efficacy, locus of control, and the theory of planned behavior 1. Journal of applied social psychology, 32, 665-683.

Azjen, I. (1980). Understanding attitudes and predicting social behavior. Englewood Cliffs.

Bandura, A., \& Walters, R.H. (1977). Social learning theory: Prentice-hall Englewood Cliffs, NJ.

Becker, M.H. (1974). The health belief model and sick role behavior. Health education monographs, 2, 409-419.

Bentler, P. M., \& Chou, C. P. (1987). Practical issues in structural modeling. Sociological methods \& research, 16(1), 78-117.

Betsch, C., Böhm, R., Korn, L., \& Holtmann, C. (2017). On the benefits of explaining herd immunity in vaccine advocacy. Nature human behaviour, 1, 1-6.

Bloom, B.R., Nowak, G.J., \& Orenstein, W. (2020). “When Will We Have a Vaccine?”Understanding Questions and Answers about Covid-19 Vaccination. New England Journal of Medicine.

Brabin, L., Roberts, S. A., Farzaneh, F., \& Kitchener, H. C. (2006). Future acceptance of adolescent human papillomavirus vaccination: a survey of parental attitudes. Vaccine, 24(16), 3087-3094.

Brewer, N.T., \& Fazekas, K.I. (2007). Predictors of HPV vaccine acceptability: a theoryinformed, systematic review. Preventive medicine, 45, 107-114. 
Brewer, N. T., Chapman, G. B., Gibbons, F. X., Gerrard, M., McCaul, K. D., \& Weinstein, N. D. (2007). Meta-analysis of the relationship between risk perception and health behavior: the example of vaccination. Health Psychology, 26, 136.

Brien, S., Kwong, J.C., \& Buckeridge, D.L. (2012). The determinants of 2009 pandemic A/H1N1 influenza vaccination: a systematic review. Vaccine, 30, 1255-1264.

Catalano, H.P., Knowlden, A.P., Birch, D.A., Leeper, J.D., Paschal, A.M., \& Usdan, S.L. (2017). Using the theory of planned behavior to predict HPV vaccination intentions of college men. Journal of American College Health, 65, 197-207.

Cates, J.R., Brewer, N.T., Fazekas, K.I., Mitchell, C.E., \& Smith, J.S. (2009). Racial differences in HPV knowledge, HPV vaccine acceptability, and related beliefs among rural, southern women. The Journal of Rural Health, 25, 93-97.

Centers for Disease Control and Prevention (2011). Ten great public health achievements-United States, 2001-2010. MMWR. Morbidity and mortality weekly report, 60, 619.

Chapman, G.B., \& Coups, E.J. (1999). Predictors of influenza vaccine acceptance among healthy adults. Preventive medicine, 29, 249-262.

Chen, M.-F., Wang, R.-H., Schneider, J.K., Tsai, C.-T., Jiang, D.D.-S., Hung, M.-N., et al. (2011). Using the health belief model to understand caregiver factors influencing childhood influenza vaccinations. Journal of Community Health Nursing, 28, 29-40.

Dryhurst, S., Schneider, C.R., Kerr, J., Freeman, A.L., Recchia, G., Van Der Bles, A.M., et al. (2020). Risk perceptions of COVID-19 around the world. Journal of Risk Research, 1-13.

Dubé, E., Laberge, C., Guay, M., Bramadat, P., Roy, R., \& Bettinger, J.A. (2013). Vaccine hesitancy: an overview. Human vaccines \& immunotherapeutics, 9, 1763-1773. 
Fishbein, M., \& Ajzen, I. (2011). Predicting and changing behavior: The reasoned action approach: Taylor \& Francis.

Gargano, L.M., Painter, J.E., Sales, J.M., Morfaw, C., Jones, L.M., Murray, D., et al. (2011). Seasonal and 2009 H1N1 influenza vaccine uptake, predictors of vaccination, and selfreported barriers to vaccination among secondary school teachers and staff. Human Vaccines, 7, 89-95.

Gerend, M.A., \& Shepherd, J.E. (2012). Predicting human papillomavirus vaccine uptake in young adult women: comparing the health belief model and theory of planned behavior. Annals of Behavioral Medicine, 44, 171-180.

Gerend, M.A., Shepherd, M.A., \& Shepherd, J.E. (2013). The multidimensional nature of perceived barriers: Global versus practical barriers to HPV vaccination. Health psychology, 32, 361 .

Groves, S. (2020, December 4). Data shows Americans couldn't resist Thanksgiving travel. $A P$ NEWS. https://apnews.com/article/data-americans-thanksgiving-travel209e88a889664ad5e25a81ee0d46201c

Guidry, J.P., Carlyle, K.E., Perrin, P.B., LaRose, J.G., Ryan, M., \& Messner, M. (2019). A path model of psychosocial constructs predicting future Zika vaccine uptake intent. Vaccine, $37,5233-5241$.

Hu, L.t., \& Bentler, P.M. (1999). Cutoff criteria for fit indexes in covariance structure analysis: Conventional criteria versus new alternatives. Structural equation modeling: a multidisciplinary journal, 6, 1-55. 
Janz, N. K., \& Becker, M. H. (1984). The health belief model: A decade later. Health education quarterly, 11(1), 1-47.

Kline, R.B. (2015). Principles and practice of structural equation modeling: Guilford publications.

Korkmaz, S., Goksuluk, D., \& Zararsiz, G. (2014). MVN: An R package for assessing multivariate normality. The R Journal, 6, 151-162.

Kumar, S., Quinn, S.C., Kim, K.H., Musa, D., Hilyard, K.M., \& Freimuth, V.S. (2012). The social ecological model as a framework for determinants of 2009 H1N1 influenza vaccine uptake in the United States. Health education \& behavior, 39, 229-243.

Larson, H.J., Jarrett, C., Eckersberger, E., Smith, D.M., \& Paterson, P. (2014). Understanding vaccine hesitancy around vaccines and vaccination from a global perspective: a systematic review of published literature, 2007-2012. Vaccine, 32, 2150-2159.

Lipschitz, J.M., Fernandez, A.C., Larson, H.E., Blaney, C.L., Meier, K.S., Redding, C.A., et al. (2013). Validation of decisional balance and self-efficacy measures for HPV vaccination in college women. American Journal of Health Promotion, 27, 299-307.

Liu, S., Yang, J.Z., \& Chu, H. (2019). When we increase fear, do we dampen hope? Using narrative persuasion to promote human papillomavirus vaccination in China. Journal of Health Psychology, 1359105319894626.

Liu, S., Yang, J.Z., Chu, H., Sun, S., \& Li, H. (2018). Different culture or different mind? Perception and acceptance of HPV vaccine in China and in the US. Journal of Health Communication, 23, 1008-1016.

Lloyd, R. (2020, September 25). Coronavirus News Roundup: September 19-September 25. Scientific American. Retrieved at: 
https://www.scientificamerican.com/article/coronavirus-news-roundup-september-19september-25/

MacDonald, N.E. (2015). Vaccine hesitancy: Definition, scope and determinants. Vaccine, 33, 4161-4164.

Maloney, E.K., Lapinski, M.K., \& Witte, K. (2011). Fear appeals and persuasion: A review and update of the extended parallel process model. Social and Personality Psychology Compass, 5, 206-219.

Marlow, L.A., Waller, J., \& Wardle, J. (2007). Trust and experience as predictors of HPV vaccine acceptance. Human Vaccines, 3, 171-175.

Muthén, L., \& Muthén, B. (2018). Mplus. The comprehensive modelling program for applied researchers: user's guide, 5.

Nan, X. (2012). Communicating to young adults about HPV vaccination: Consideration of message framing, motivation, and gender. Health Communication, 27, 10-18.

Noar, S.M., \& Zimmerman, R.S. (2005). Health behavior theory and cumulative knowledge regarding health behaviors: are we moving in the right direction? Health education research, 20, 275-290.

Palan, S., \& Schitter, C. (2018). Prolific. ac-A subject pool for online experiments. Journal of Behavioral and Experimental Finance, 17, 22-27.

Petrovic, K., Burney, S., \& Fletcher, J. (2011). The relationship of knowledge, health value and health self-efficacy with men's intentions to receive the human papillomavirus (HPV) vaccine. Journal of Health Psychology, 16, 1198-1207. 
Qian, D., \& Li, O. (2020). The Relationship between Risk Event Involvement and Risk Perception during the COVID-19 Outbreak in China. Applied Psychology: Health and Well-Being

Quinn, S. C., Jamison, A. M., An, J., Hancock, G. R., \& Freimuth, V. S. (2019). Measuring vaccine hesitancy, confidence, trust and flu vaccine uptake: Results of a national survey of White and African American adults. Vaccine, 37(9), 1168-1173.

Quinn, S.C., Jamison, A., Freimuth, V.S., An, J., Hancock, G.R., \& Musa, D. (2017). Exploring racial influences on flu vaccine attitudes and behavior: Results of a national survey of White and African American adults. Vaccine, 35, 1167-1174.

Rimal, R. N., \& Real, K. (2003). Perceived risk and efficacy beliefs as motivators of change: Use of the risk perception attitude (RPA) framework to understand health behaviors. Human communication research, 29(3), 370-399.

Schoch-Spana, M., Brunson, E., Long, R., Ravi, S., Ruth, A., \& Trotochaud, M. (2020). The public's role in Covid-19 vaccination: planning recommendations informed by design thinking and the social, behavioral, and communication sciences. Baltimore: Johns Hopkins Center for Health Security.

Shahrabani, S., \& Benzion, U. (2012). How experience shapes health beliefs: the case of influenza vaccination. Health education \& behavior, 39, 612-619.

Sidi, Y., \& Harel, O. (2018). The treatment of incomplete data: reporting, analysis, reproducibility, and replicability. Social Science \& Medicine, 209, 169-173.

Slovic, P., Finucane, M.L., Peters, E., \& MacGregor, D.G. (2004). Risk as analysis and risk as feelings: Some thoughts about affect, reason, risk, and rationality. Risk Analysis: An International Journal, 24, 311-322. 
Smith, P.J., Humiston, S.G., Marcuse, E.K., Zhao, Z., Dorell, C.G., Howes, C., et al. (2011). Parental delay or refusal of vaccine doses, childhood vaccination coverage at 24 months of age, and the Health Belief Model. Public health reports, 126, 135-146.

Stout, M. E., Christy, S. M., Winger, J. G., Vadaparampil, S. T., \& Mosher, C. E. (2020). Selfefficacy and HPV Vaccine Attitudes Mediate the Relationship Between Social Norms and Intentions to Receive the HPV Vaccine Among College Students. Journal of community health.

Tyson, A., Johnson, C., \& Funk, C. (2020). U.S. Public Now Divided Over Whether To Get COVID-19 Vaccine: Concerns about the safety and effectiveness of possible vaccine, pace of approval process.

Visser, O., Hulscher, M.E., Antonise-Kamp, L., Akkermans, R., van der Velden, K., Ruiter, R.A., et al. (2018). Assessing determinants of the intention to accept a pertussis cocooning vaccination: A survey among healthcare workers in maternity and paediatric care. Vaccine, $36,736-743$.

Weinstein, N.D. (1993). Testing four competing theories of health-protective behavior. Health psychology, 12, 324.

Westland, J. C. (2010). Lower bounds on sample size in structural equation modeling. Electronic commerce research and applications, 9(6), 476-487.

Witte, K. (1994). Fear control and danger control: A test of the extended parallel process model (EPPM). Communications Monographs, 61, 113-134.

World Health Organization. (2020). Coronavirus disease (COVID-19) Weekly Epidemiological Update and Weekly Operational Update. Retrived at: https://www.who.int/emergencies/diseases/novel-coronavirus-2019/situation-reports 
Xiao, X., \& Borah, P. (2020). Do Norms Matter? Examining Norm-Based Messages in HPV Vaccination Promotion. Health Communication, 1-9. 


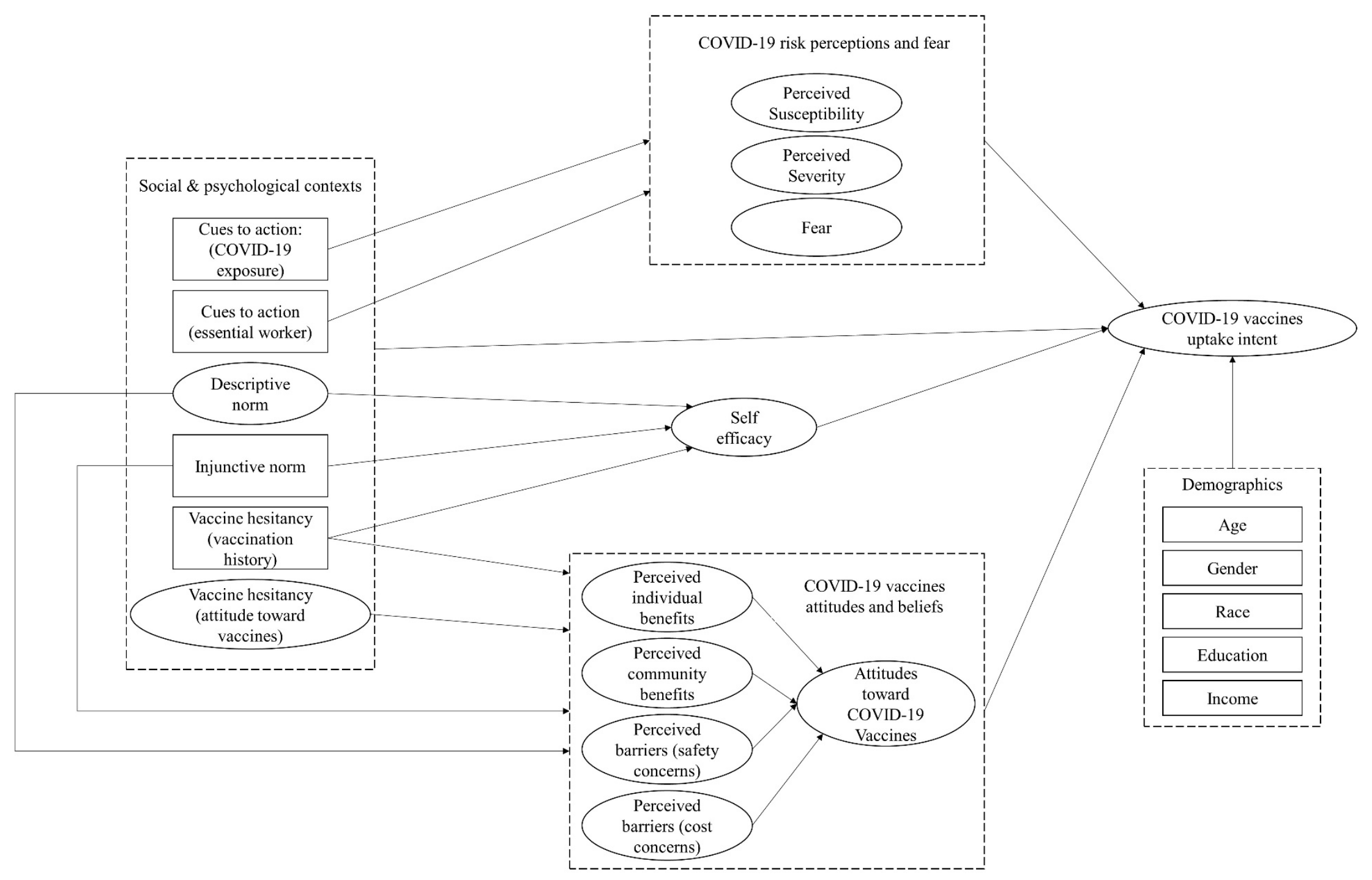

Figure 1. Theoretical framework 


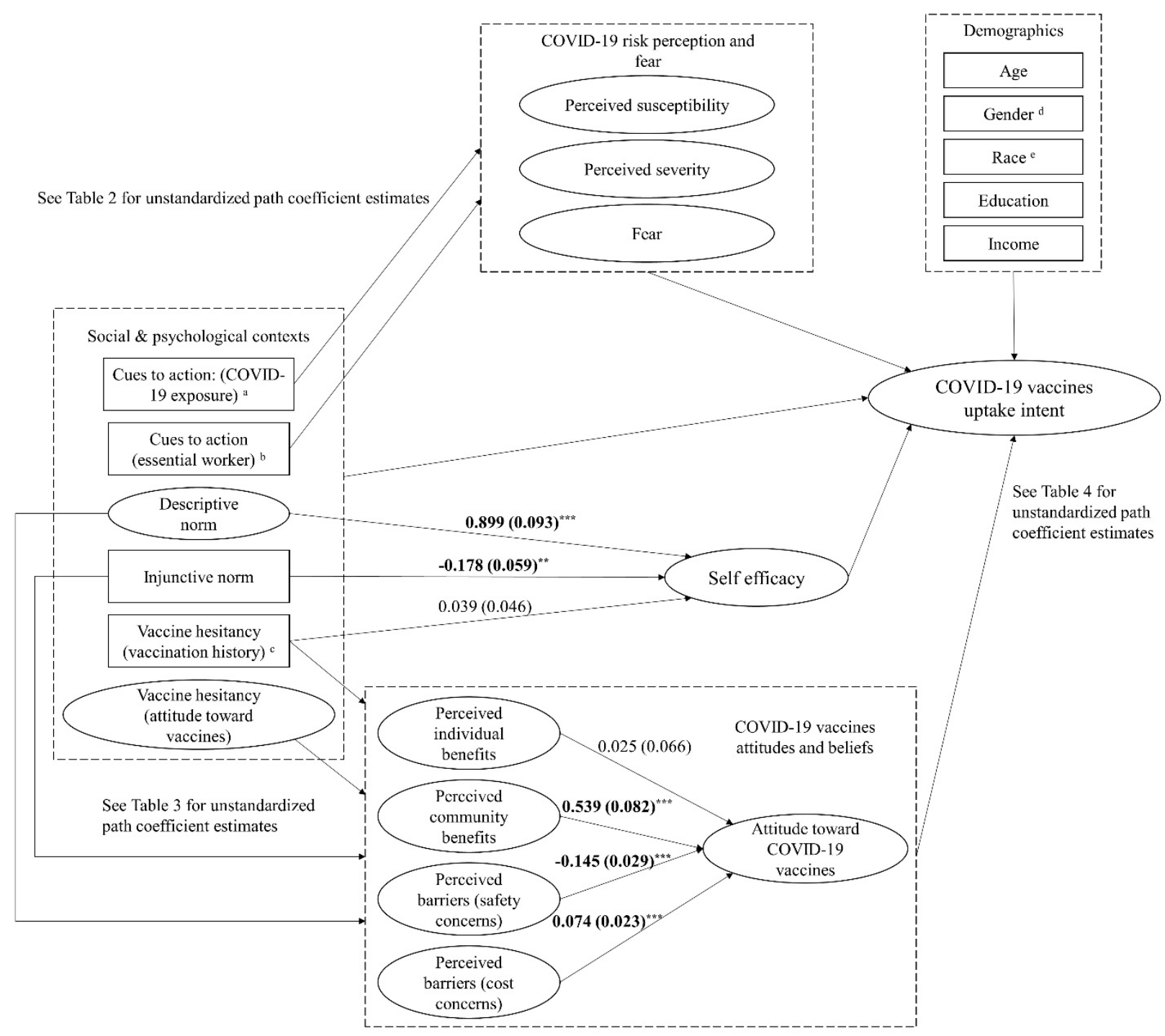

Figure 2. Unstandardized path coefficient estimates $(B(S E))$

Note. ${ }^{\text {a }}$ Dummy-coded variable $(1=$ knowing someone who was tested positive for or died due to COVID$19,0=$ not having such experience);

${ }^{\mathrm{b}}$ dummy-coded variable $(1=$ served as an essential worker during COVID-19 lockdowns; $0=$ did not serve as an essential worker during COVID-19 lockdowns);

${ }^{\mathrm{c}}$ dummy-coded variable $(1=$ received vaccines in the past 18 months, $0=$ did not receive vaccine in the past 18 months or not sure);

d dummy-coded variable with male as reference group;

${ }^{\mathrm{e}}$ dummy-coded variable with non-Hispanic White or Caucasian as reference group;

${ }^{*} p<.05 ;{ }^{* *} p<.01 ;{ }^{* * *} p<.001$. 


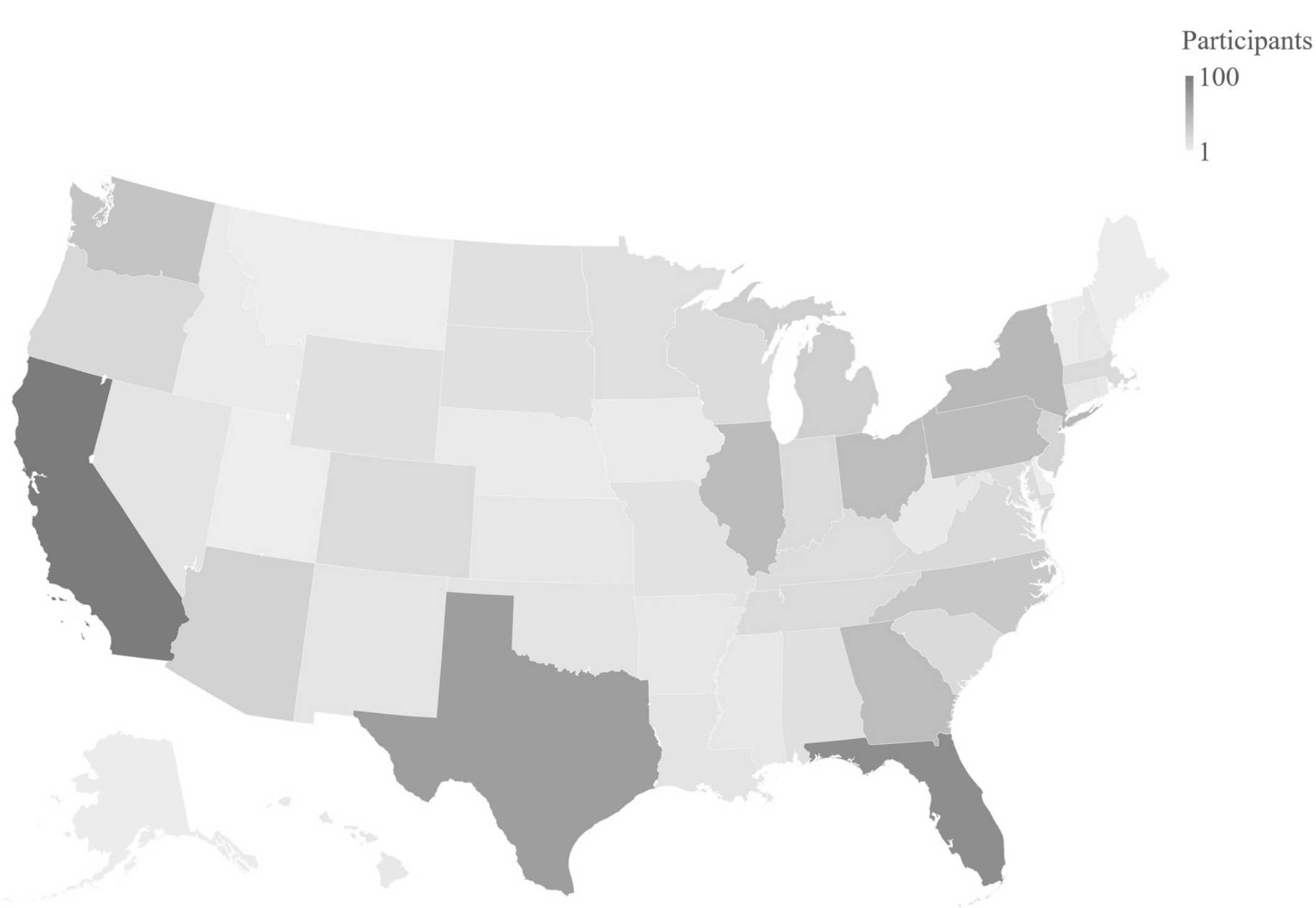

Figure 3. Geographical distribution of survey participants 
Table 1

Descriptive statistics and composite reliability of survey measures

M SD Reliability

\section{COVID-19 risk perception and fear}

Perceived susceptibility to COVID-19

It is likely that I will get COVID-19. (1 "strongly disagree" to 5 "strongly agree")

I am at risk of getting COVID-19.

It is possible that I will get COVID-19.

Perceived severity of COVID-19

I believe that COVID-19 is a severe health problem. (1 "strongly disagree" to 5 "strongly agree")

I believe that COVID-19 has serious negative consequences.

I believe that COVID-19 is extremely harmful.

Fear

On a scale of 1 (not at all) to 5 (very much), please indicate how you feel when you think about COVID-19: Fear

... Afraid

... Scared.

\subsection{5}

.78

$\alpha=.74$

$2.77 \quad .88$

$3.54 \quad 1.04$

$3.75 \quad .94$

$4.21 \quad .89 \quad \alpha=.92$

$4.24 \quad .99$

$4.29 \quad .87$

$4.12 \quad 1.00$

2.68

1.33

$\alpha=0.96$

$2.71 \quad 1.37$

$2.66 \quad 1.41$

$2.67 \quad 1.395$

\section{COVID-19 vaccines attitudes and beliefs}

\section{Attitude toward COVID-19 vaccines}

Using the following adjective scales, please indicate how much you feel that getting vaccinated for COVID-19 is: 1 "Negative" to 5 "Positive"

... 1 "Unfavorable" to 5 "Favorable"
$4.16 \quad 1.19 \quad \alpha=0.98$

$4.40 \quad 1.04$

$4.38 \quad 1.08$ 
... 1 "Bad" to 5 "Good"

... 1 "Harmful" to 5 "Beneficial"

... 1 "Foolish" to 5 "Wise"

Perceived individual benefits of COVID-19 vaccines

COVID-19 vaccines will work in preventing the disease. (1 "strongly disagree" to 5 "strongly agree")

COVID-19 vaccines will be effective in preventing COVID-19.

If I get the vaccines, I will be less likely to get COVID-19.

Perceived community benefits of COVID-19 vaccines

Having myself vaccinated against COVID-19 is beneficial for the health of others in my community. (1 "strongly disagree" to 5 "strongly agree")

COVID-19 vaccines protect the health of my community.

Perceived barriers of getting COVID-19 vaccines (safety concerns)

How much would the following factors prevent you from getting vaccinated for COVID-19: Concerns about whether COVID-19 vaccines are safe $(1=$ not at all, $5=$ a great deal $)$

Not enough research done on COVID-19 vaccines

I have concerns about possible side effects of COVID-19 vaccines

Perceived barriers of getting COVID-19 vaccines (cost concerns)

How much would the following factors prevent you from getting vaccinated for COVID-19: Vaccine cost (it's too expensive) (1 "not at all" to 5 "a great deal")

I'm not sure how to file the insurance claim to get reimbursed

My insurance may not cover COVID-19 vaccine
$4.44 \quad 1.01$

$4.41 \quad 1.02$

$4.45 \quad 1$

$3.75 \quad 0.86 \quad \alpha=0.91$

$3.71 \quad 0.92$

$3.64 \quad 0.89$

$3.89 \quad 0.99$

$4.10 \quad 0.94 \quad r=0.85$

$4.13 \quad 1.01$

$4.08 \quad 0.96$

$3.31 \quad 1.23 \quad \alpha=0.88$

$3.39 \quad 1.38$

$3.36 \quad 1.36$

$3.18 \quad 1.37$

$2.01 \quad 1.07 \quad \alpha=0.80$

$2.32 \quad 1.38$

$1.72 \quad 1.11$

$1.98 \quad 1.28$ 


\begin{tabular}{|c|c|c|c|}
\hline Self-efficacy & 3.5 & 0.76 & $\alpha=0.77$ \\
\hline $\begin{array}{l}\text { I will be able to get the vaccines to prevent contracting COVID-19. (1 } \\
\text { "strongly disagree" to } 5 \text { "strongly agree") }\end{array}$ & 3.67 & 0.85 & \\
\hline $\begin{array}{l}\text { It will be easy for me to get the vaccines to protect myself from } \\
\text { COVID-19. }\end{array}$ & 3.42 & 0.86 & \\
\hline Getting vaccinated to prevent COVID-19 is convenient. & 3.43 & 1.02 & \\
\hline
\end{tabular}

\section{Social and psychological contexts}

Cues to action (COVID-19 exposure)

Have you experienced any of the following in the past 9 months?

Tested positive for COVID-19 (excluded from final sample)

$n=10$

Tested positive for COVID-19 antibody (excluded from final sample)

$$
n=3
$$

Hospitalized due to COVID-19 (excluded from final sample)

$$
n=1
$$

Family or close friend tested positive for COVID-19

Someone you knew tested positive for COVID-19

$0.37(n=342)$

Exposed to people who have got COVID-19

$0.08(n=73)$

Treating patients with COVID-19

$0.01(n=12)$

Taking care of someone with COVID-19

$0.01(n=9)$

Family or close friend passed away due to COVID-19

$0.05(n=46)$

Someone you knew passed away due to COVID-19

$0.12(n=115)$

None of above

$0.50(n=464)$

Cues to action (served as essential worker)

Were you an essential worker during the COVID-19 lockdown? (1 "yes", 0 "no") 
Subjective norm (descriptive norm)

Most people who are like me will get vaccinated for COVID-19. (1

"strongly disagree", 5 "strongly agree")

Most people who are important to me will get vaccinated for COVID19.

Subjective norm (injunctive norm) Most people who are important to me think that I should get COVID-19 vaccines (1 "strongly disagree", 5

"strongly agree")

Baseline vaccine hesitancy (attitude toward vaccines in general)

Using the following adjective scales, please indicate how much you feel that getting vaccines is: 1 "Negative" to 5 "Positive"

... 1 "Unfavorable" to 5 "Favorable"

... 1 "Bad" to 5 "Good"

... 1 "Harmful" to 5 "Beneficial"

... 1 "Foolish" to 5 "Wise"

Baseline vaccine hesitancy (vaccination history). Have you received any vaccine such as flu shot in the past 18 months? - Yes

No

Not sure

COVID-19 vaccines uptake intent

One the scale of 1 (very unlikely) to 5 (very likely), please indicate the likelihood that you will: Consider getting COVID-19 vaccines.

... Try to get COVID-19 vaccines.

... Actually get vaccinated for COVID-19.

... Get vaccinated if a physician offered you COVID-19 vaccines.
3.69

$0.9 \quad r=0.66$

3.66

0.99

$3.71 \quad 0.98$

3.67

1.07

4.42

0.99

$\alpha=0.98$

$4.40 \quad 1.04$

$4.38 \quad 1.08$

$4.44 \quad 1.01$

$4.41 \quad 1.02$

$4.45 \quad 1$

$0.51(n=480)$

$0.47(n=441)$

$0.02(n=13)$

$3.98 \quad 1.25 \quad \alpha=0.98$

$4.15 \quad 1.25$

$3.88 \quad 1.32$

$3.86 \quad 1.31$

$4.02 \quad 1.29$


Table 2

Unstandardized coefficients estimate for paths predicting perceived susceptibility to, perceived severity and fear of COVID-19

\begin{tabular}{|c|c|c|c|}
\hline & Perceived susceptibility & Perceived severity & Fear \\
\hline & $B(S E)$ & $B(S E)$ & $B(S E)$ \\
\hline Cues to action (exposure) & $0.218(0.041)^{* * *}$ & $0.257(0.063){ }^{* * *}$ & $0.376(0.085))^{* * *}$ \\
\hline Cues to action (essential worker) & $0.134(0.051){ }^{* *}$ & $-0.046(0.08)$ & $-0.216(0.107) *$ \\
\hline
\end{tabular}

Note. ${ }^{*} p<.05 ;{ }^{* *} p<.01 ;{ }^{* * *} p<.001$; significant path estimates are in boldface; outcome variables are specified in the first row 
Table 3

Unstandardized coefficient estimates for paths predicting perceived individual and community benefits and perceived safety and const concerns of COVID-19 vaccines and attitudes toward COVID-19 vaccines

\begin{tabular}{|c|c|c|c|c|c|}
\hline $\begin{array}{l}\text { Vaccine hesitancy } \\
\text { (attitude toward vaccines) }\end{array}$ & $0.273(0.041)^{* * * *}$ & $0.423(0.044)^{* * *}$ & $-0.129(0.062) *$ & $0.143(0.059)$ * & $0.471(0.056){ }^{* * *}$ \\
\hline Injunctive norm & $-0.079(0.051)$ & $0.03(0.05)$ & $-0.003(0.08)$ & $0.016(0.089)$ & $0.07(0.045)$ \\
\hline
\end{tabular}

Note. ${ }^{*} p<.05 ;{ }^{* *} p<.01 ;{ }^{* * *} p<.001 ;$ significant path estimates are in boldface; outcome variables are specified in the first row 
Table 4

Unstandardized coefficient estimates for paths predicting COVID-19 vaccines uptake intent

\begin{tabular}{lc}
\hline \\
\hline Contextual ches)
\end{tabular}

Contextual cues and baseline vaccine hesitancy

Vaccine hesitancy (attitude toward vaccine)

$0.113(0.043)^{* * *}$

Vaccine hesitancy (vaccination history)

$0.144(0.044)^{* * *}$

Injunctive norm

$0.046(0.058)$

Descriptive norm

$0.410(0.174)^{*}$

Cues to action (exposure)

$0.048(0.039)$

Cues to action (essential worker)

$0.030(0.051)$

Risk perception and fear

Perceive susceptibility

$0.114(0.062)$

Perceived severity

$0.002(0.040)$

Fear

$0.050(0.018)^{* * *}$

Beliefs about COVID-19 vaccines

Perceived individual benefits

$0.044(0.085)$

Perceived community benefits

$0.228(0.089)^{* *}$

Perceived barriers (safety concerns)

$-0.118(0.027)^{* * * *}$

Perceived barriers (cost concerns)

$0.009(0.026)$

Attitudes toward COVID-19 vaccines

$0.276(0.044)^{* * * *}$

Self-efficacy

Self-efficacy

Demographics

Age

$0.001(0.001)$

Female $^{1}$

$-0.071(0.037)$ 
Other gender ${ }^{1}$

Black or African American ${ }^{2}$

Hispanic or Latino ${ }^{2}$

Asian, Pacific Islander, and Native American ${ }^{2}$

Other racial groups ${ }^{2}$

Education

Income
$0.001(0.128)$

$-0.116(0.061)$

$-0.099(0.082)$

$0.088(0.061)$

$0.017(0.135)$

$0.009(0.013)$

$-0.004(0.01)$

Note. ${ }^{1}$ dummy-coded variable with male as reference group $;{ }^{2}$ dummy-coded variable with nonHispanic White or Caucasian as reference group; ${ }^{*} p<.05 ;{ }^{* *} p<.01 ;{ }^{* * *} p<.001$; ; significant path estimates are in boldface 
Table 5

Total direct and indirect effects of contextual factors and COVID-19 vaccines attitudes and beliefs on vaccine uptake intent

\begin{tabular}{|c|c|c|c|c|c|c|}
\hline & \multicolumn{2}{|c|}{ Total effect } & \multicolumn{2}{|c|}{ Total indirect effect } & \multicolumn{2}{|c|}{ Direct effect } \\
\hline & Estimate & $95 \% \mathrm{CI}^{1}$ & Estimate & $95 \% \mathrm{CI}^{1}$ & Estimate & $95 \% \mathrm{CI}^{1}$ \\
\hline \multicolumn{7}{|c|}{ COVID-19 vaccines attitudes and beliefs } \\
\hline $\begin{array}{c}\text { Cues to action }{ }^{3} \\
\text { (COVID-19 exposure) }\end{array}$ & 0.092 & {$[0.013,0.170]$} & 0.044 & {$[0.013,0.075]$} & 0.048 & {$[-0.033,0.128]$} \\
\hline $\begin{array}{l}\text { Cues to action }{ }^{4} \\
\text { (essential worker) }\end{array}$ & 0.034 & {$[-0.074,0.142]$} & 0.005 & {$[-0.024,0.033]$} & 0.030 & {$[-0.079,0.138]$} \\
\hline $\begin{array}{l}\text { Vaccine hesitancy } \\
\text { (vaccination history) }\end{array}$ & 0.117 & {$[0.018,0.216]$} & -0.027 & {$[-0.084,0.03]$} & 0.144 & {$[0.054,0.234]$} \\
\hline $\begin{array}{c}\text { Vaccine hesitancy } \\
\text { (attitude toward vaccines) }\end{array}$ & 0.440 & {$[0.340,0.539]$} & 0.327 & {$[0.235,0.42]$} & 0.113 & {$[0.024,0.201]$} \\
\hline \multicolumn{7}{|l|}{ Social and psychological contexts } \\
\hline Descriptive norm & 0.614 & {$[0.385,0.844]$} & 0.205 & {$[-0.106,0.515]$} & 0.410 & {$[-0.038,0.858]$} \\
\hline Injunctive norm & 0.102 & {$[-0.022,0.225]$} & 0.056 & {$[-0.027,0.138]$} & 0.046 & {$[-0.097,0.189]$} \\
\hline Perceived individual benefits & 0.051 & {$[-0.136,0.238]$} & 0.007 & {$[-0.031,0.045]$} & 0.044 & {$[-0.138,0.226]$} \\
\hline $\begin{array}{l}\text { Perceived community } \\
\text { benefits }\end{array}$ & 0.376 & {$[0.180,0.572]$} & 0.149 & {$[0.078,0.219]$} & 0.228 & {$[0.032,0.424]$} \\
\hline $\begin{array}{l}\text { Perceived barriers } \\
\text { (safety concerns) }\end{array}$ & -0.158 & {$[-0.215,-0.101]$} & -0.040 & {$[-0.061,-0.019]$} & -0.118 & {$[-0.174,-0.062]$} \\
\hline $\begin{array}{l}\text { Perceived barriers } \\
\text { (cost concerns) }\end{array}$ & 0.029 & {$[-0.025,0.084]$} & 0.020 & {$[0.007,0.034]$} & 0.009 & {$[-0.045,0.063]$} \\
\hline
\end{tabular}


HBT \& COVID-19 VACCINE

Appendix

Supplementary Table 1

Unstandardized coefficient estimates for paths predicting COVID-19 vaccines uptake intent in the multiplicative model which include the interaction between perceived severity and susceptibility

$B(S E)$

Contextual cues and baseline vaccine hesitancy

Vaccine hesitancy (attitude toward vaccine)

$0.123(0.044)^{* * *}$

Vaccine hesitancy (vaccination history)

$0.138(0.044)^{* * *}$

Injunctive norm

$0.042(0.058)$

Descriptive norm

$0.41(0.173)^{*}$

Cues to action (exposure)

$0.047(0.039)$

Cues to action (essential worker)

$0.027(0.051)$

Risk perception and fear

Perceive susceptibility

$0.124(0.061)$

Perceived severity

$0.012(0.039)$

Perceived susceptibility $\times$ perceived severity

$0.062(0.035)$

Fear

$0.046(0.017)^{* *}$

Beliefs about COVID-19 vaccines

Perceived individual benefits

$0.056(0.085)$

Perceived community benefits

$0.226(0.089)^{*}$

Perceived barriers (safety concerns)

$-0.118(0.027)^{* * * *}$

Perceived barriers (cost concerns)

$0.01(0.026)$

Attitudes toward COVID-19 vaccines

$0.269(0.044)^{* * *}$

Self-efficacy

Self-efficacy

$-0.158(0.119)$ 
HBT \& COVID-19 VACCINE

Demographics

Age

$0.001(0.001)$

Female $^{1}$

$-0.074(0.037)^{*}$

Other gender ${ }^{1}$

$-0.007(0.129)$

Black or African American ${ }^{2}$

$-0.111(0.061)$

Hispanic or Latino ${ }^{2}$

$-0.089(0.081)$

Asian, Pacific Islander, and Native American ${ }^{2}$

$0.09(0.062)$

Other racial groups ${ }^{2}$

$0.037(0.131)$

Education

$0.007(0.013)$

Income

$-0.003(0.01)$

Note. ${ }^{1}$ dummy-coded variable with male as reference group ${ }^{2}$ dummy-coded variable with nonHispanic White or Caucasian as reference group; ${ }^{*} p<.05 ;{ }^{* *} p<.01 ;{ }^{* * *} p<.001$; ; significant path estimates are in boldface 